\title{
Osteoclast-secreted CTHRC1 in the coupling of bone resorption to formation
}

\author{
Sunao Takeshita, ${ }^{1}$ Toshio Fumoto, ${ }^{1}$ Kazuhiko Matsuoka, ${ }^{1}$ Kyoung-ae Park, ${ }^{1}$ \\ Hiroyuki Aburatani, ${ }^{2}$ Shigeaki Kato, ${ }^{3}$ Masako Ito, ${ }^{4}$ and Kyoji Ikeda ${ }^{1}$
}

\begin{abstract}
1Department of Bone and Joint Disease, National Center for Geriatrics and Gerontology (NCGG), Obu, Aichi, Japan. ${ }^{2}$ Genomescience Division, RCAST, University of Tokyo School of Medicine, Tokyo, Japan. ${ }^{3}$ nnstitute of Molecular Cellular Bioscience, University of Tokyo, Tokyo, Japan. ${ }^{4}$ Medical Work-Life-Balance Center, Nagasaki University Hospital, Nagasaki, Japan.
\end{abstract}

\begin{abstract}
Bone remodeling is characterized by the sequential, local tethering of osteoclasts and osteoblasts and is key to the maintenance of bone integrity. While bone matrix-mobilized growth factors, such as TGF- $\beta$, are proposed to regulate remodeling, no in vivo evidence exists that an osteoclast-produced molecule serves as a coupling factor for bone resorption to formation. We found that CTHRC1, a protein secreted by mature boneresorbing osteoclasts, targets stromal cells to stimulate osteogenesis. Cthrc1 expression was robustly induced when mature osteoclasts were placed on dentin or hydroxyapatite, and also by increasing extracellular calcium. Cthrc1 expression in bone increased in a high-turnover state (such as that induced by RANKL injections in vivo), but decreased in conditions associated with suppressed bone turnover (such as with aging and after alendronate treatment). Targeted deletion of $C t h r c 1$ in mice eliminated $C t h r c 1$ expression in bone, whereas its deficiency in osteoblasts did not exert any significant effect. Osteoclast-specific deletion of $C t h r c 1$ resulted in osteopenia due to reduced bone formation and impaired the coupling process after resorption induced by RANKL injections, impairing bone mass recovery. These data demonstrate that CTHRC1 is an osteoclastsecreted coupling factor that regulates bone remodeling.
\end{abstract}

\section{Introduction}

Bone is constantly remodeled through the removal of old bone (resorption) and the replacement of new bone (formation) by hematopoietic-derived osteoclasts and mesenchymal-derived osteoblasts, respectively, to meet structural and metabolic demands $(1,2)$. It has long been believed that a preceding resorption phase is a prerequisite for the initiation of subsequent bone formation (3), which is also supported by recent clinical observations that treatment of osteoporotic patients with the potent antiresorptive drug alendronate blunts the anabolic action of parathyroid hormone (PTH) (4). Although the coupling of bone formation to resorption has been long recognized, the mechanism and the factors mediating this fundamental process in skeletal homeostasis are not fully understood (5).

Recently, it was suggested that active TGF- $\beta 1$ released from bone matrix during bone resorption is coupled to bone formation by inducing migration of marrow stromal cells to resorption sites (6). Bidirectional signaling between EPHRINB2 on osteoclasts and the receptor EPHB4 on osteoblasts has also been proposed to link bone resorption and formation through direct cell-cell contact (7). In addition, mature osteoclasts produce and secrete factors, such as WNT10B, BMP6, and the lipid mediator sphingosine-1-phosphate (S1P), that have been shown to stimulate osteoblast recruitment and survival (8-10). However, the in vivo function of these factors in the coupling process remains to be elucidated.

We reasoned that a factor mediating the coupling reaction linking bone resorption to formation would have to meet the following criteria: (a) it should be produced and secreted/presented by osteoclasts; (b) it should exert an effect on osteoblastic differentia-

Conflict of interest: The authors have declared that no conflict of interest exists. Citation for this article: J Clin Invest. 2013;123(9):3914-3924. doi:10.1172/JCI69493. tion and/or function, thereby promoting bone formation; (c) in its absence, the coupling of bone formation to preceding resorption should be impaired; and (d) its expression or activity should be regulated by agents or conditions known to affect bone turnover, such as aging and alendronate treatment. We have now identified a protein product of mature osteoclasts that fulfills these criteria and is considered to be a novel coupling factor.

\section{Results}

Identification of Cthrc1 in bone-resorbing active, but not inactive, osteoclasts. In an effort to identify an osteoclast-derived coupling factor, we performed gene expression analysis during osteoclast differentiation using mouse genome arrays containing 39,000 transcripts, and found a transcript of the collagen triple helix repeat containing 1 (Cthrc1) gene. This transcript was absent in bone marrow macrophages (BMMs) and preosteoclasts (pOCs) and only modestly expressed in multinucleated mature osteoclasts prepared on plastic dishes, but was markedly induced in mature osteoclasts prepared on dentin slices, i.e., functional osteoclasts that are actively engaged in bone resorption (Supplemental Figure 1; supplemental material available online with this article; doi:10.1172/ JCI69493DS1). This was confirmed by RT-PCR (Figure 1A); notably, calcitonin receptor (Calcr), a known product of mature osteoclasts, was expressed in inactive mature osteoclasts prepared on plastic dishes, whereas Cthrc1 mRNA expression was robustly induced in mature osteoclasts prepared on dentin slices (Figure 1B). This suggests that Cthrc1 expression in osteoclasts is tightly associated with bone resorption.

Cthrc1 was originally isolated as a gene induced after balloon injury of the rat aorta (11); it is not expressed in arteries under physiological conditions (Figure 1C and ref. 11) and is thought to play a role in vascular remodeling in response to injury by regulating TGF- $\beta$ responsiveness (12). It was also shown to be responsive 
A
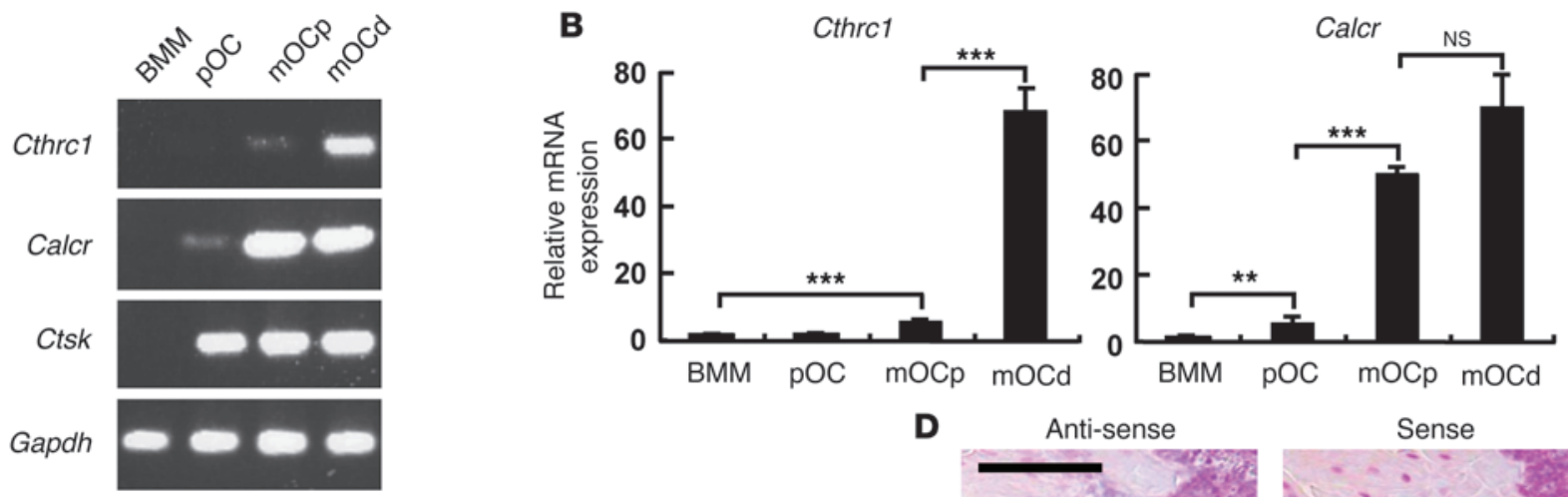

C
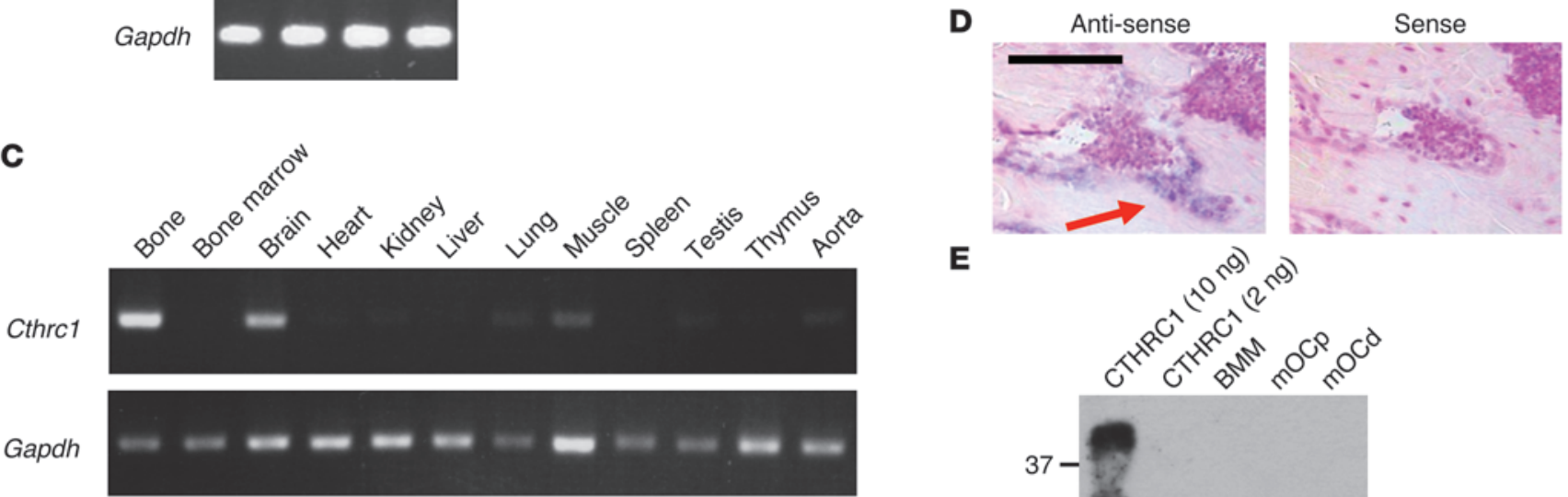

$\mathbf{E}$

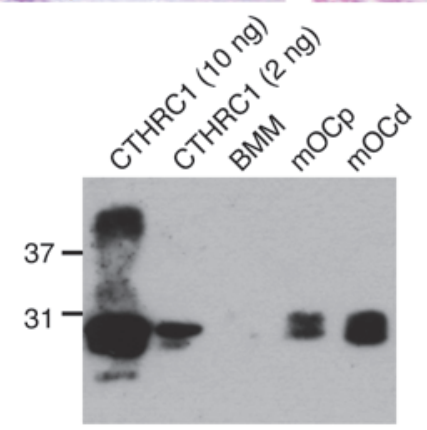

Figure 1

CTHRC1 is a secretory product of active, bone-resorbing osteoclasts. (A and B) Induction of Cthrc1 mRNA in mature osteoclasts on dentin actively engaged in bone resorption. Cthrc1, Calcr, and Ctsk mRNA expression was analyzed in BMMs, in pOCs, and in mature osteoclasts placed on dentin slices (mOCd) or on plastic dishes (mOCp) by RT-PCR (A) and quantitative RT-PCR (B). $n=3$ per group. (C) Tissue distribution of Cthrc1 mRNA by RT-PCR. Cthrc1 was mainly expressed in bone and brain, but not in normal (uninjured) aorta. (D) The Cthrc1 transcript (blue) in multinucleated osteoclasts on trabecular bone (arrow) in vivo, determined by ISH. Sense Cthrc1 probe served as the negative control. Scale bar: $100 \mu \mathrm{m}$. (E) Mature osteoclasts secreted CTHRC1 protein. CTHRC1 protein was detected in the conditioned medium of mature osteoclasts by immunoblot after immunoprecipitation. Recombinant CTHRC1 protein served as a positive control. ${ }^{* \star} P<0.01 ;{ }^{* *} P<0.001$.

to TGF- $\beta$ in chondrocytes $(13,14)$. In fact, we confirmed by in situ hybridization (ISH) that during development, Cthrc1 transcript was prominently expressed in chondrocytes (Supplemental Figure 2). However, the tissue distribution pattern observed in adult mice indicated that $C t h r c 1$ was expressed in bone, and to a much lesser extent in brain, but not in any other tissue (Figure 1C). In the skeletal tissue, the Cthrc1 transcript was observed in multinucleated osteoclasts on the bone surface in vivo, but not in osteoblasts or osteocytes (as shown by ISH), and it colocalized with TRAP staining (Figure 1D and Supplemental Figure 3, A and B). Thus, we conclude that the major source of CTHRC1 in the adult skeleton is bone-resorbing osteoclasts.

Murine Cthrc1 encodes a 245-amino acid protein with a leader sequence of 32 amino acids, and the amino acid sequence is highly conserved among the mouse, rat, and human proteins (mouse/ rat, 99.2\%; mouse/human, 97.7\%; rat/human, 95.7\%). CTHRC1 shares a collagen domain containing 12 Gly-X-X repeats and a globular domain in the $\mathrm{C}$-terminal half with adiponectin and is thought to belong to the adiponectin/complement factor family (Supplemental Figure 4A). Recombinant mouse CTHRC1 was generated using a baculovirus/insect cell system and purified to near homogeneity (data not shown), which was then used to raise a polyclonal antibody. This antibody detected CTHRC1 protein in bone-resorbing osteoclasts cultured on a dentin slice (Supplemental Figure 3C). Western blot analysis revealed that the recombinant protein migrates as a single polypeptide of apparently $28 \mathrm{kDa}$ under reducing conditions, while several bands with higher molecular weights were observed under nonreducing conditions (Supplemental Figure 4B), which suggests that CTHRC1 exists in monomeric, dimeric, and trimeric forms, just like adiponectin (15). The antibody detected a protein of approximately $28 \mathrm{kDa}$ in the conditioned medium of mature osteoclasts, but not in the conditioned medium of BMMs, and the detected amount increased substantially in mature osteoclasts prepared on dentin slices (Figure 1E), supporting our contention that CTHRC1 is a secretory product of mature, functional osteoclasts.

Regulation of Cthrc1 gene expression by contact with bydroxyapatite and calcium. In order to gain further insight into the mechanism of Cthrc1 gene expression in osteoclasts, mature osteoclasts generated from BMMs ex vivo with M-CSF and RANKL were placed on different substrates, including a plastic dish, a dentin slice, or a hydroxyapatite (HA) disc. RT-PCR analysis revealed that Cthrc1 was even more robustly induced on HA than on dentin slices (Figure 2A). Accordingly, increasing extracellular calcium concen- 

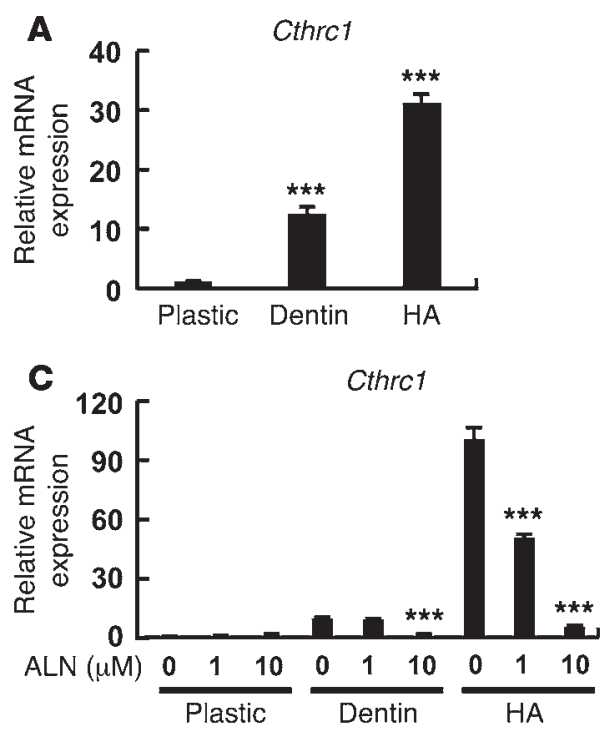

Figure 2

Regulation of Cthrc1 gene expression. (A) Cthrc1 mRNA expression was strongly induced in osteoclasts cultured on HA. Osteoclasts were cultured on plastic plates, dentin slices, or Osteologic discs composed of HA crystals, and RNAs were prepared. Real-time RT-PCR for Cthrc1 was performed. (B) Increasing extracellular calcium concentrations increased Cthrc1 transcription in osteoclasts on plastic dishes. Osteoclasts were cultured at the indicated $\mathrm{CaCl}_{2}$ concentrations for 24 hours. (C) Alendronate (ALN) inhibited the Cthrc1 expression induced in osteoclasts cultured on dentin or HA. (D) Proton pump inhibitors or calcitonin inhibited Cthrc 1 expression. Osteoclasts were cultured on different substrates (plastic plates, dentin slices, or $\mathrm{HA}$ discs) in the presence of $100 \mathrm{nM}$ bafilomycin $\mathrm{A} 1$ (BM), $1 \mu \mathrm{M}$ N-ethylmaleimide (NEM), or $1 \mathrm{nM}$ calcitonin (CT). $n=3 .{ }^{*} P<0.05 ;{ }^{* \star} P<0.01 ;{ }^{* * *} P<0.001$.

trations, as well as phosphate, stimulated Cthrc1 mRNA expression even in inactive mature osteoclasts prepared on plastic dishes (Figure 2B and Supplemental Figure 5). Other divalent cations, such as magnesium and gadolinium, had only modest effects (Supplemental Figure 5).

The upregulated Cthrc1 expression in mature osteoclasts cultured on HA or dentin was markedly and dose-dependently inhibited when bone resorption was inhibited by alendronate, by the vacuolar $\mathrm{H}^{+}$ATPase (V-ATPase) inhibitors bafilomycin A1 or $\mathrm{N}$-ethylmaleimide, and by the direct osteoclast inhibitor calcitonin (Figure 2, C and D). Collectively, these data suggest that mineral ions such as $\mathrm{Ca}^{2+}$ and $\mathrm{PO}_{4}{ }^{3-}$ are important regulators of Cthrc1 expression in mature osteoclasts and that activation of the bone resorption machinery, especially V-ATPase, is a prerequisite of CTHRC1 production.

Cthrc1 stimulates osteoblast differentiation. In order to define the function of CTHRC1 in osteoblastogenesis, Cthrc1 was retrovirally expressed in calvaria-derived primary osteoblastic cells. Quantitative RT-PCR analysis revealed that forced Cthrc1 expression stimulated osteogenic differentiation, as demonstrated by increased alkaline phosphatase $(A l p)$ and osteocalcin (Bglap) expression at day 7 and 14 after confluency (Figure 3A). Osteogenic induction was confirmed by ALP activity and mineralization, as determined by alizarin red staining (Figure 3B). Treatment of bone marrow stromal ST2 cells with the recombinant CTHRC1 protein also increased ALP activity (Figure 3C). CTHRC1 also suppressed adipogenic marker expression (Figure 3A) and inhibited adipocytic differentiation, as determined by Oil Red-O staining (Figure 3D). face receptor in order to stimulate osteoblastic differentiation as well as recruitment, thereby promoting bone formation.

Conditional Cthrc1 KO in osteoclasts. In order to gain insight into the physiologic function of osteoclast-derived CTHRC1 in bone remodeling, we generated mice carrying a floxed $C t h r c 1$ allele (Figure 4A). The floxed mice were then mated with Ctsk-Cre (17) or CAG-Cre (18) mice to generate osteoclast-specific conditional Cthrc1 KO ( $\triangle \mathrm{OC})$ or systemic Cthrc1 KO (sKO) mice, respectively (Supplemental Figure 7A). Both $\triangle \mathrm{OC}$ and sKO mice were born with the expected Mendelian frequency, appeared grossly normal, grew normally, and were fertile (data not shown). Specific deletion of Cthrc1 in osteoclasts was verified in mature osteoclasts generated ex vivo (Supplemental Figure 7B). Quantitation of Cthrc1 mRNA expression in bone by RT-PCR demonstrated its near-complete absence not only in sKO bone, but also in $\triangle \mathrm{OC}$ bone, with no difference between them (Figure $4 \mathrm{~B}$ ), which indicates that mature osteoclasts are the major source of CTHRC1 in bone in vivo.

$\mu \mathrm{CT}$ scanning of the proximal tibial metaphysis revealed that $\triangle \mathrm{OC}$ and sKO mice exhibited a low bone mass phenotype with microstructural abnormalities reminiscent of osteoporosis, such as decreased trabecular number and thickness (Figure 4C and data not shown). Histomorphometric analysis at the proximal tibial metaphysis revealed that osteoid surface and bone formation rate were significantly decreased in $\triangle \mathrm{OC}$ versus control mice, while the bone resorption parameters (eroded surface and osteoclast surface) did not differ between them (Figure 4D). These results suggest that osteoclast-produced CTHRC1 carries out an important physiological function in the maintenance of bone mass and trabecular structure, mainly through regulation of bone formation. 

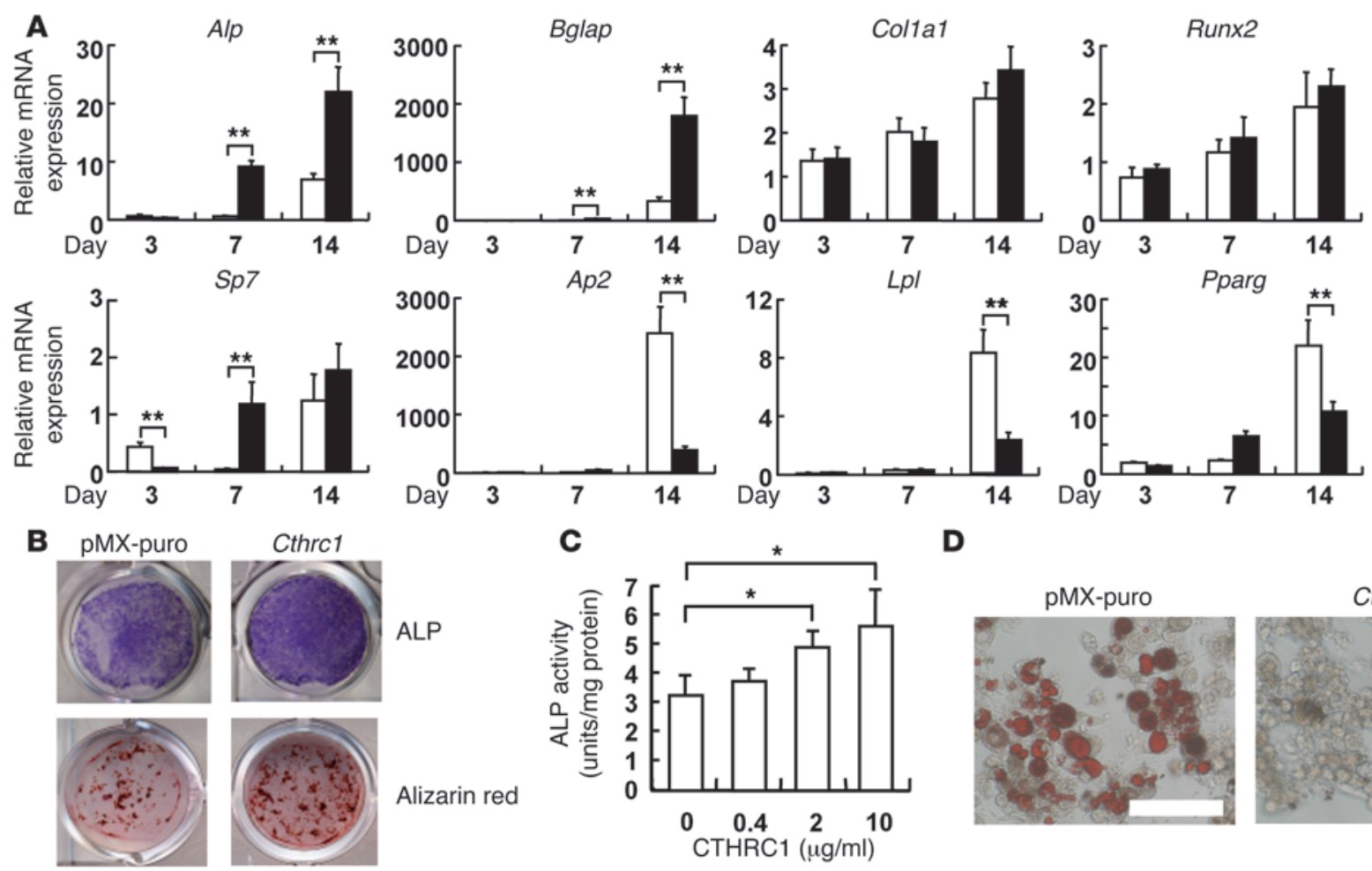

E

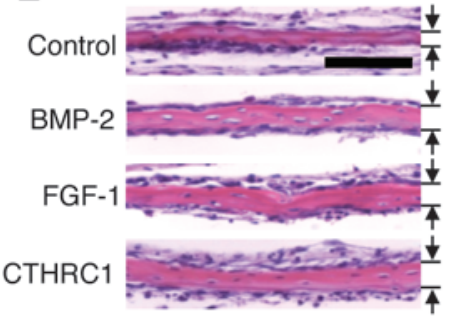

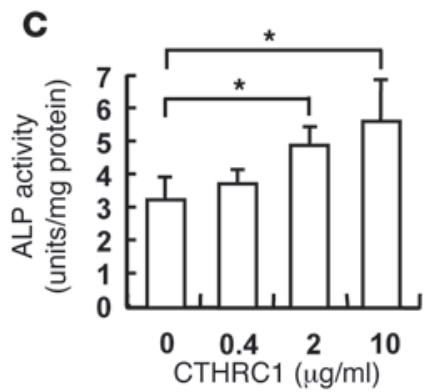

D

ALP
Alizarin red
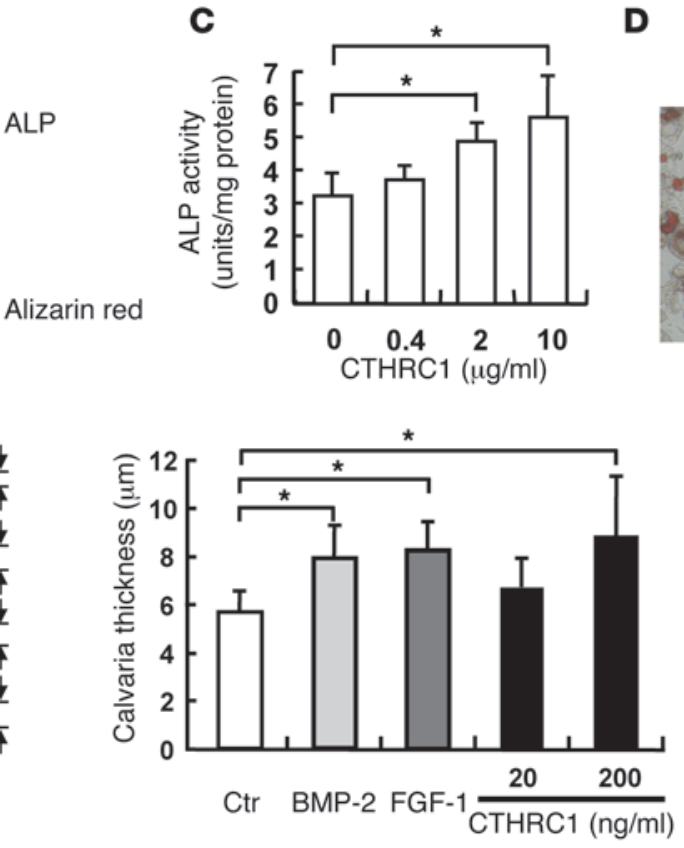
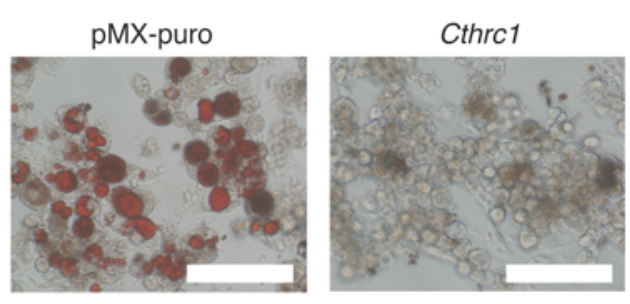

$\mathbf{F}$

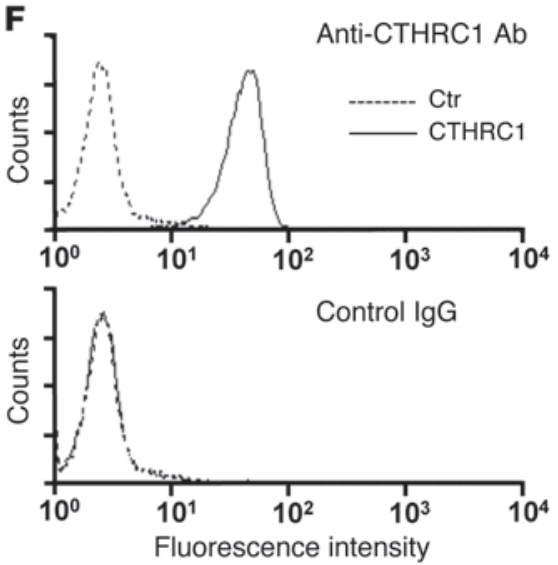

Figure 3

CTHRC1 stimulates osteoblastogenesis and bone formation. (A) Retroviral transduction with Cthrc1 stimulated osteoblastogenesis and suppressed adipogenesis, as determined by gene expression. Calvarial osteoblastic cells were transduced with pMX-puro vector (white bars) or pMX-puro-Cthrc 1 (black bars). On days 3, 7, and 14 after cells reached confluence, RNAs were extracted, and quantitative RT-PCR analysis was performed for expression of Alp, Bglap, Col1a1, Runx2, and Sp7 for osteoblastogenesis and Ap2, Lpl, and Pparg for adipogenesis. See Supplemental Table 1 for primers. $n=3$. (B) Cells were stained for ALP and Alizarin red on days 7 and 14 , respectively. (C) Recombinant mouse CTHRC1 protein stimulated ALP activity in ST2 cells dose dependently. $n=3$. (D) CTHRC1 inhibited adipocyte differentiation from 3T3-L1 cells, as determined by Oil Red-O staining. Scale bar: $100 \mu \mathrm{m}$. (E) CTHRC1 stimulated bone formation in organ culture. Mouse calvaria was treated with recombinant CTHRC1 (20 and $200 \mathrm{ng} / \mathrm{ml})$, BMP-2 (100 ng/ml), or FGF-1 (100 ng/ml), and bone-forming activity was assessed by measuring calvaria thickness (arrows). $n=3$. Scale bars: $100 \mu \mathrm{m}$. (F) Binding of CTHRC1 to a putative receptor on ST2 cells. After treatment of ST2 cells with recombinant CTHRC1 protein (solid line) or PBS control (dashed line), cells were stained with rabbit anti-CTHRC1 polyclonal antibody (top) or rabbit IgG as a negative control (bottom) and PE-conjugated goat anti-rabbit IgG, then analyzed using flow cytometry. ${ }^{*} P<0.05$; ${ }^{\star \star} P<0.01$.

Coupling function in vivo. In order to address the functional importance of CTHRC1 in the coupling process from bone resorption to formation, we developed a mouse model using recombinant RANKL injections (Figure 5A). In this model, robust stimulation of bone resorption by the osteoclast differentiation factor RANKL reportedly takes place as early as $2-3$ days (19). $\mu$ CT analysis further revealed that the $3 \mathrm{D}$ bone volume (BV) decreased rapidly, reaching a nadir at 2 weeks, followed by gradual recovery to baseline at 8 weeks, presumably due to the subsequent stimulation of bone formation (Figure 5B). We interpret these dynamic changes in bone mass to mean that the catabolic phase of the first 1-2 weeks after RANKL injections triggered subsequent stimula- 
A

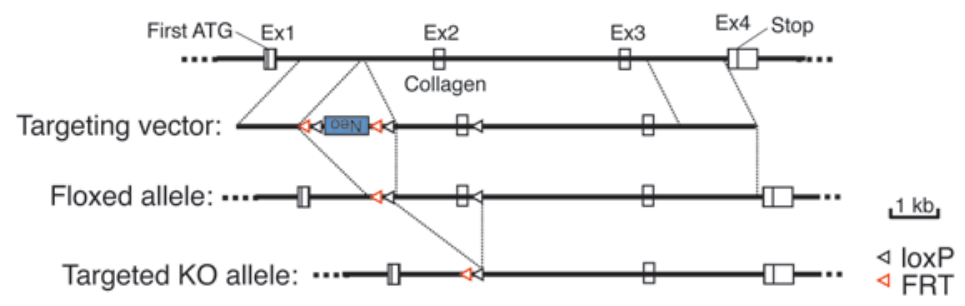

B

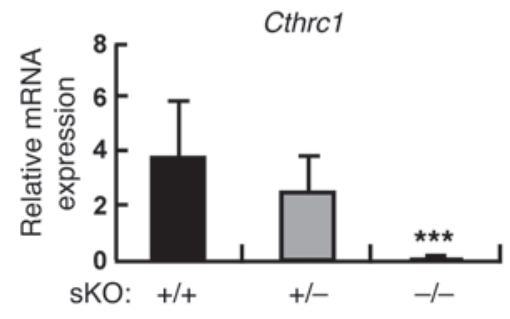

C
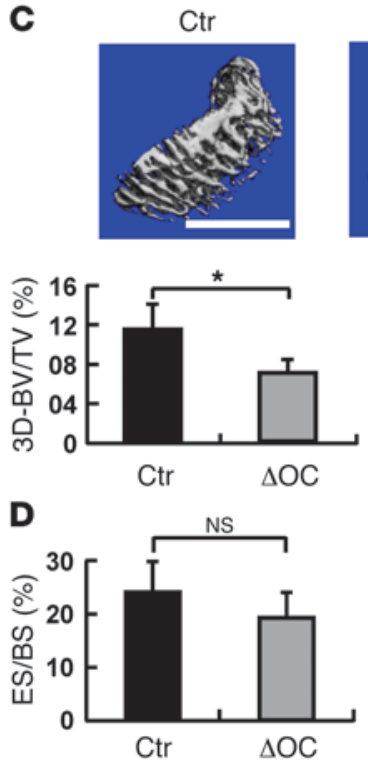

$\triangle \mathrm{OC}$

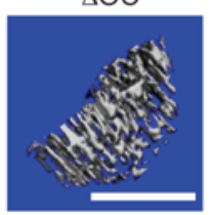

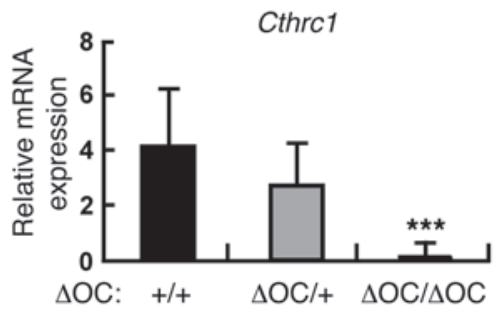
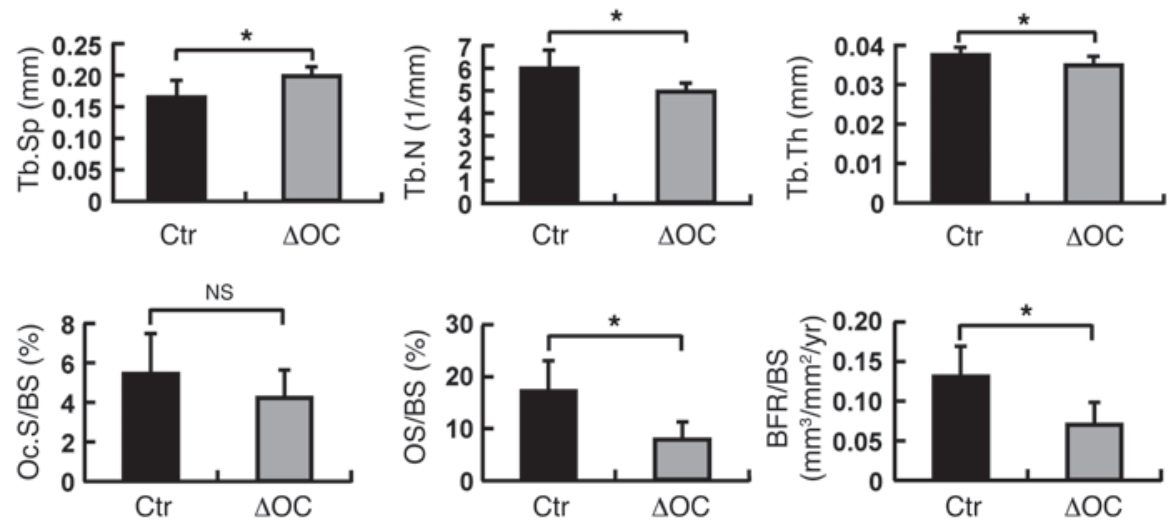

Figure 4

Osteoclast-specific Cthrc1 KO results in a low bone mass phenotype with reduced bone formation. (A) Design of the floxed allele of $C$ thrc1. (B) Comparable reduction of Cthrc1 expression in bone of $\triangle \mathrm{OC}$ and sKO mice (generated by crossing Cthrc $1^{\mathrm{fl} / \mathrm{fl}}$ mice with Ctsk-Cre and CAG-Cre mice, respectively). (C) Reduced $3 \mathrm{D}$-determined $\mathrm{BV}$ relative to tissue volume (3D-BV/TV) and microstructural derangements, by $\mu C T$ scanning, in $\triangle \mathrm{OC}$ versus Cthrc $1^{f l / f l}$ control mice. Representative 3D images of proximal tibia are also shown. Tb.N, trabecular number; Tb. Th, trabecular thickness; Tb.Sp, trabecular separation. Scale bars: $1 \mathrm{~mm} . n=5$. (D) Reduced bone formation parameters by histomorphometry in $\Delta$ OC mice. Osteoid surface (OS), bone formation rate (BFR), eroded surface (ES), and osteoclast surface (Oc.S) were corrected for bone surface (BS). ${ }^{\star} P<0.05$; ${ }^{\star \star \star} P<0.001$.

tion of bone formation, resulting in a net transient decrease in bone mass at 2 weeks followed by the anabolic phase and complete recovery at 8 weeks.

Cthrc1 expression in bone was markedly increased, together with that of osteoclast markers such as tartrate-resistant acid phosphatase (Acp5), Calcr, and cathepsin $\mathrm{K}$ (Ctsk), in control mice 3 days after RANKL administration compared with PBS injection (Figure 6A). In $\triangle \mathrm{OC}$ mice, induction of Cthrc1 expression after RANKL injections, as well as basal Cthrc1 expression, was suppressed almost completely, whereas expression of the mature osteoclast molecular markers Calcr and Ctsk was increased to the same extent as in control mice (Figure 6A). In fact, the degree of bone loss at 10 days and urinary CTX excretion at 2 days after RANKL injections was indistinguishable from that of the control group
(Figure 6, B and C), which indicates that the presence or absence of CTHRC1 made no difference in the initial phase of osteoclast activation and bone loss induced by RANKL injection. However, the recovery of bone mass was significantly impaired in $\triangle \mathrm{OC}$ versus control mice (Figure 6B), and this was associated with significantly reduced osteoblast recruitment and matrix synthesis and significant impairment of bone formation rate and lower serum osteocalcin concentrations in $\triangle \mathrm{OC}$ mice (Figure 6, D and E).

Conditional Cthrc $1 \mathrm{KO}$ in osteoblasts. In contrast to $\triangle \mathrm{OC}$ mice, specific Cthrc1 deletion in osteoblasts by crossing the floxed mouse with the Osx1-Cre mouse ( $\triangle \mathrm{OB}$ mice; Figure 7A and ref. 20 ) had no significant effect on either basal Cthrc1 expression in bone or its upregulation after RANKL injections (Figure 7B), which confirmed that osteoclasts, but not osteoblasts, are the 

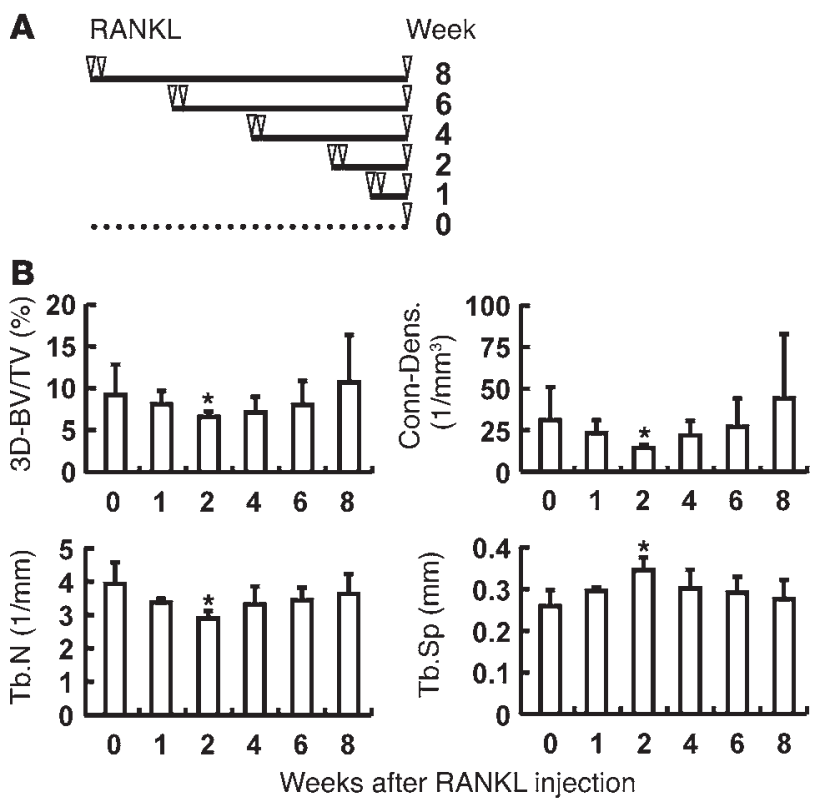

Figure 5

RANKL injection model to assess coupling function in vivo. (A) After 2 s.c. injections of RANKL (triangles), mice were sacrificed at 1, 2, 4, 6, and 8 weeks. (B) $\mu \mathrm{CT}$ analysis of the trabecular region of the proximal tibial metaphysis. Conn-Dens., connectivity density. $n=3$ per group. ${ }^{*} P<0.05$.

major source of CTHRC1 in adult bone. This was further supported by the finding that $\triangle \mathrm{OB}$ mice exhibited normal bone mass (Figure 7, C and D). Taken together, these results suggest that CTHRC1 produced by mature osteoclasts is required for an adequate anabolic response after the catabolic phase induced by RANKL injections, providing in vivo evidence that the protein has physiologic function in the process coupling bone resorption to formation.

Correlation of Cthrc1 expression with bone turnover states. Finally, in order to determine whether CTHRC1 plays any pathogenic role in age-related bone loss, we examined whether its expression changes with aging. Comparison of $\mathrm{mRNA}$ expression in bone among mice of different ages revealed that Cthrc1 expression declined substantially with age in both males and females (Figure 8A and data not shown). Alendronate, a potent inhibitor of bone turnover, is widely used for treatment of high-turnover bone disease, such as postmenopausal osteoporosis. However, it has been recognized clinically that inhibition of osteoclastic function by alendronate blunts the anabolic action of PTH (4), and the underlying mechanism is beginning to be understood (21). We found that along with a reduction in bone turnover markers after treatment with alendronate (Supplemental Figure 8), Cthrc1 expression in bone was substantially reduced, in contrast to the increased expression of Ctsk and Calcr (Figure 8B). These data suggest that a low bone turnover state associated with aging and alendronate treatment correlates with reduced $C t h r c 1$ expression.

Cathepsin K inhibitors have been developed as a new strategy of inhibiting bone resorption (22). Interestingly, unlike bisphosphonates, treatment with cathepsin inhibitors has not been associated with suppressed bone formation, a result that is also supported by prior reports of increased, rather than decreased, bone formation in Ctsk-deficient mice $(10,23)$. Taking advantage of the Ctsk-Cre knockin construct, we generated $C t s k^{-/-}$mice. Unlike the case with bisphosphonate, no significant reduction in Cthrc1 expression was observed in Ctsk ${ }^{-/-}$mice (Figure 8C).

Finally, as representative examples of osteoclast-poor and -rich osteopetrosis, $\mathrm{Rankl}^{-/-}$and $\mathrm{Src}^{-/-}$mice were examined (24). In accordance with the absence of osteoclasts in Rankl $^{l^{--}}$mice, almost no expression of Ctsk and Calcr was detected in Rankl ${ }^{-/-}$ bone (Figure 8D). In marked contrast, expression of both Ctsk and Calcr was markedly increased in $\mathrm{Src}^{-/}$bone (Figure 8E), consistent with the abundance of inactive osteoclasts in $\mathrm{Src}^{-/-}$bone. Importantly, Cthrc1 expression was markedly decreased in both Rankl ${ }^{-/}$ and $\mathrm{Src}^{-/-}$bone (Figure 8, D and E), consistent with our concept that the mere presence of inactive osteoclasts is not sufficient for optimal Cthrc1 expression and that the bone-resorbing activity of osteoclasts is a prerequisite for Cthrc1 expression and, therefore, for CTHRC1-mediated coupling of resorption with formation.

\section{Discussion}

It has long been recognized that in each remodeling cycle, bone formation is tightly coupled to the preceding bone resorption carried out by osteoclasts $(25,26)$. The fact that bone resorption triggers formation suggests that certain signals derived from osteoclasts recruit osteoblasts or their progenitors to the remodeling site to target bone formation activity, although it is a matter of debate whether the mere presence of osteoclasts is sufficient to initiate the coupling reaction or whether their resorptive activity is required, as well as whether mature osteoclasts or precursor cells represent the source of the coupling factor $(27,28)$. Here, we demonstrated that mature osteoclasts secreted CTHRC1 only when osteoclasts were placed on dentin or HA, which suggests that CTHRC1 production is closely linked with osteoclast attachment to calcified tissue. This situation was mimicked by placing osteoclasts in an environment high in extracellular calcium and phosphate, which indicates that recognition of these minerals by osteoclasts is essential for CTHRC1 upregulation. Furthermore, our findings that Cthrc1 expression was suppressed in osteoclasts treated with alendronate, calcitonin, or proton pump inhibitors as well as in $\mathrm{Src}^{-/}$osteoclasts in vivo suggest that establishment of polarity, cytoskeletal reorganization, and/or activation of the secretory function of acid and collagenolytic enzymes is required for CTHRC1 production. The secreted CTHRC1 protein binds to a putative cell surface receptor on stromal cells, promoting cell differentiation into mature osteoblasts and also chemotaxis along a CTHRC1 pathway, together with WNT proteins. Thus, CTHRC1 may function as a guidance molecule for targeting stromal/osteogenic cells to sites of bone resorption, thus promoting the initiation of subsequent bone-forming activity.

The physiologic importance of osteoclast-derived CTHRC1 in bone homeostasis was evidenced by the low bone mass phenotype with lowered bone formation exhibited by $\Delta \mathrm{OC}$ mice (in which Cthrc1 was specifically deleted in osteoclasts by use of Ctsk-Cre mice; ref. 17), but not by $\triangle \mathrm{OB}$ mice. Thus, CTHRC1 is a candidate molecule for mediating the coupling of bone resorption with formation. However, this does not necessarily exclude a contribution of bone matrix-derived factors, represented by TGF- $\beta 1$, as TGF- $\beta 1$ is mobilized and activated during bone resorption and promotes recruitment of osteogenic progenitors to resorption sites (6).

$\mathrm{S} 1 \mathrm{P}$, a lipid mediator that is also produced and secreted by osteoclasts, stimulates both recruitment and survival of osteoblasts $(8,9)$. $\mathrm{S} 1 \mathrm{P}$ is abundant in the circulation, and a recent report suggests that $\mathrm{S} 1 \mathrm{P}$ regulates the mobilization of osteoclast precursors to 

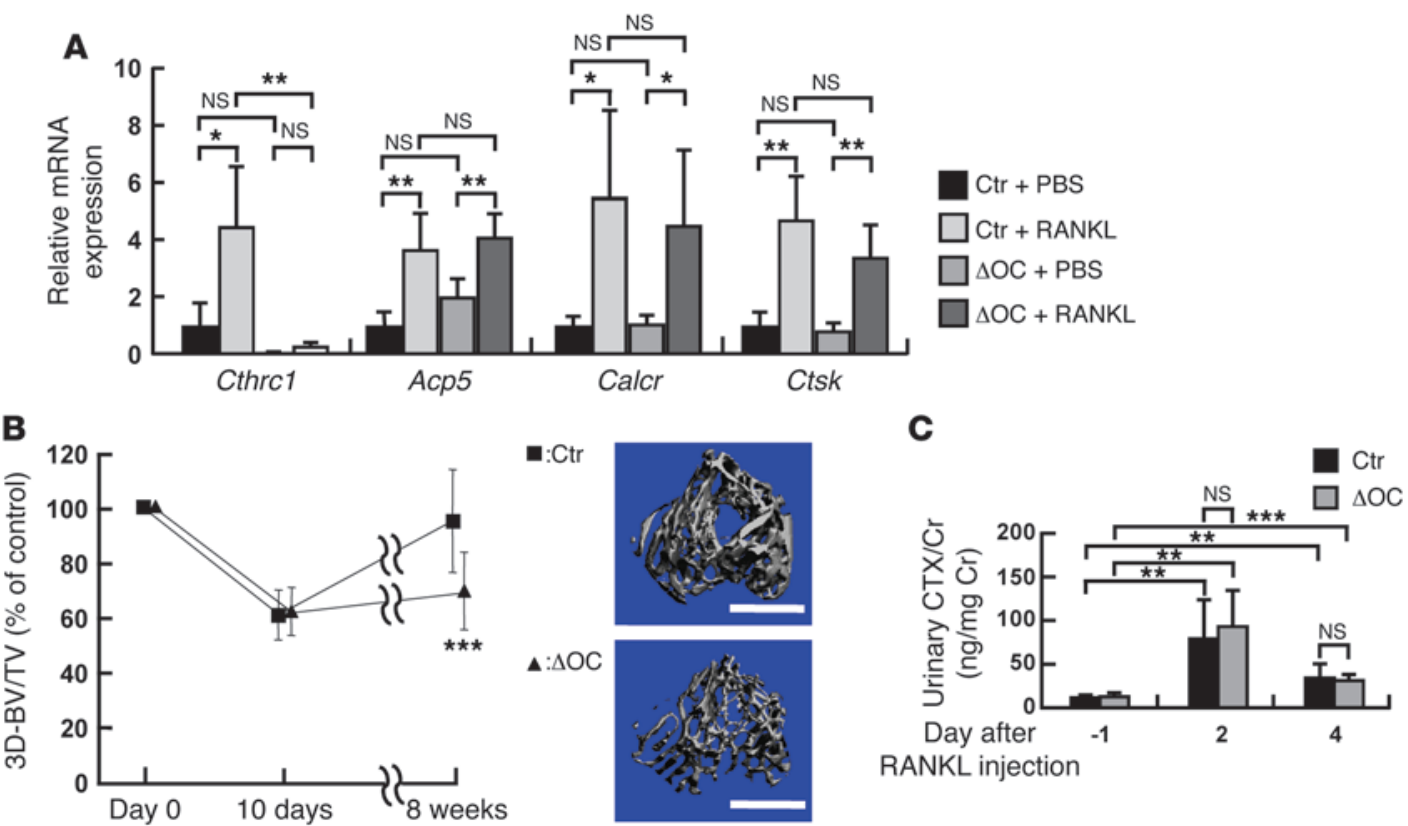

RANKL injection

D
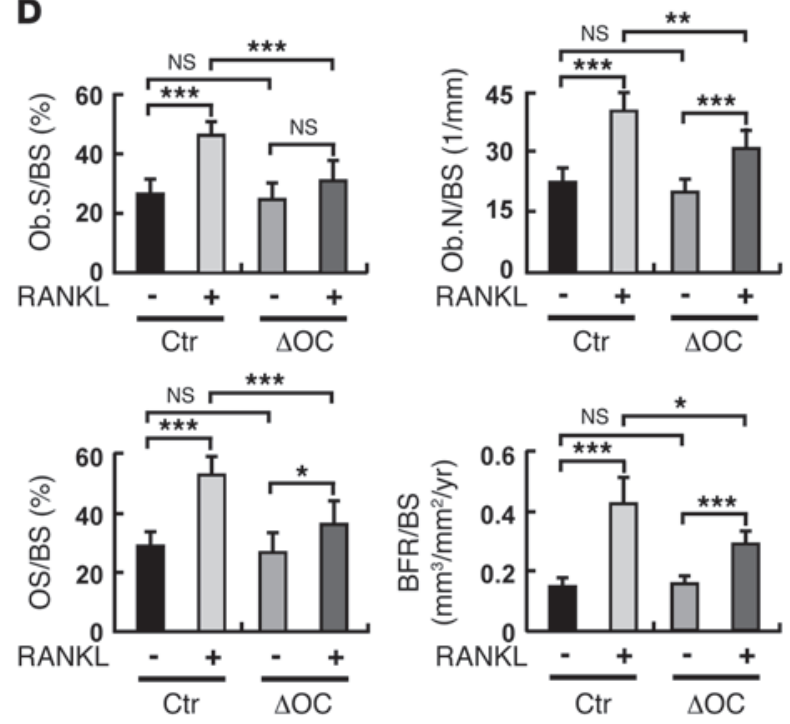

$\mathbf{E}$

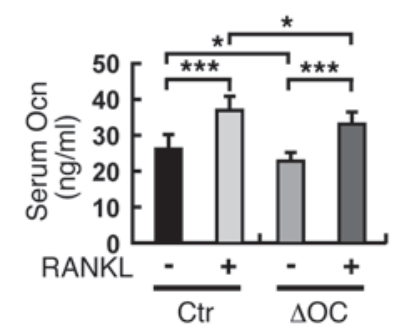

Figure 6

Impaired coupling function in osteoclast-specific Cthrc1 KO mice. (A) Cthrc1, Acp5, Calcr, and Ctsk expression at baseline and after RANKL injection in Cthrc $1^{f / f l}$ control and $\triangle \mathrm{OC}$ mice, determined by quantitative RT-PCR. Values were normalized for Gapdh. $n=4$. (B) Impaired recovery of 3D BV at 8 weeks after RANKL injection in $\triangle \mathrm{OC}$ versus Cthrc $1^{\text {fllfl }}$ control mice. Mice were sacrificed at 10 days and 8 weeks after RANKL injections, and 3D-determined BV relative to tissue volume was determined. Representative $\mu C T$ images at 8 weeks are also shown. Scale bars: $1 \mathrm{~mm}$. $n=8$. (C) Urinary CTX excretion was assessed 1 day before $(-1)$ and 2 and 4 days after RANKL injections. Data are normalized for creatinine $(\mathrm{Cr}) . n=5$. (D) Bone histomorphometric analysis was performed at 10 days after RANKL injections. Osteoblast surface (Ob.S), osteoblast number (Ob.N), osteoid surface, and bone formation rate were corrected for bone surface. $n=8$. (E) Serum osteocalcin (Ocn) concentrations were determined 10 days after RANKL injections $(n=8)$. ${ }^{\star} P<0.05 ;{ }^{* \star} P<0.01 ;{ }^{* \star \star} P<0.001$.

and from bone through the cell surface S1P receptor (29). It has recently been shown that S1P secretion is increased in Ctsk-deficient osteoclasts, which is suggested to account for the elevated bone formation in $\mathrm{Ctsk}^{-/-}$mice (10). However, the specific function of S1P and other secretory proteins, such as WNT10B and BMP6, in the coupling of formation to resorption in vivo remains elusive.

Since the coupling reaction takes place in each individual bone multicellular unit (BMU), and since bone homeostasis is maintained as a net result of numerous micron-order BMU activities throughout the entire skeleton, there is no established assay for evaluating coupling efficiency in vivo. We have attempted to synchronize osteoclast formation and bone resorption initiation by acute RANKL injections so as to separate the resorption and formation phases. It has been reported that 3 RANKL injections at 24-hour intervals induce an acute increase in bone resorption at a time point as early as 50 hours (19). We closely monitored the skeletal response to acute RANKL challenge up to 8 weeks after injection by $3 \mathrm{D} \mu \mathrm{CT}$ scanning and found that the initial catabolic pro- 
A

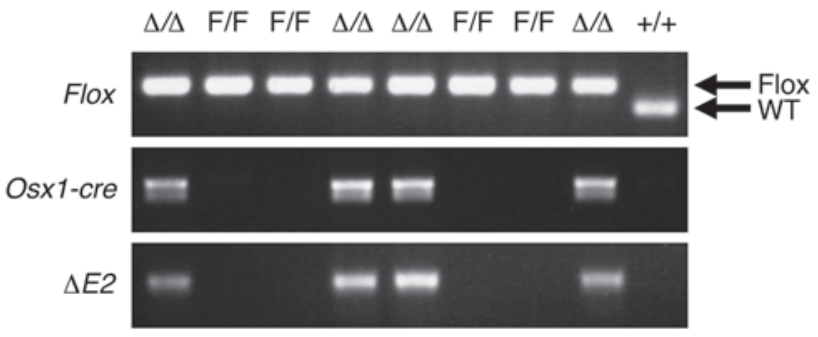

C

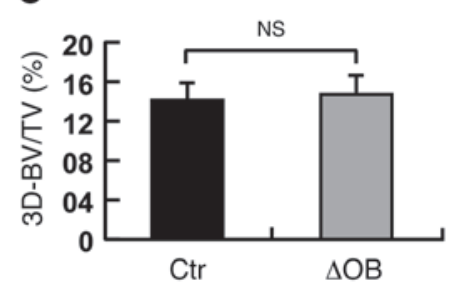

D

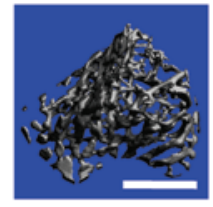

Ctr

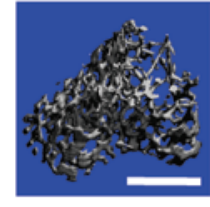

$\triangle \mathrm{OB}$

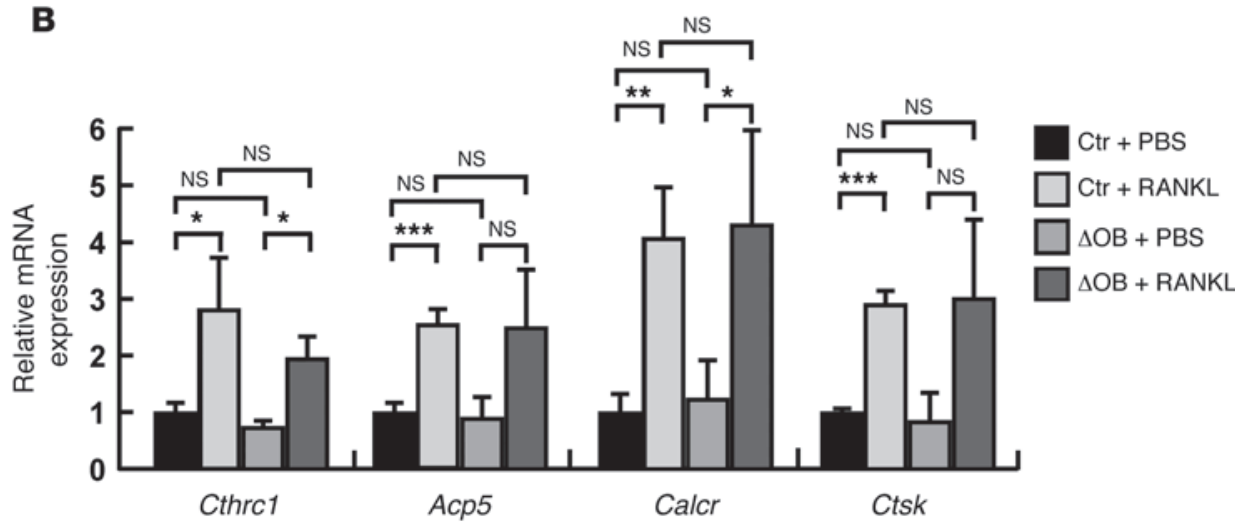

Figure 7

Osteoblast-specific Cthrc1 KO mice. (A) Genotyping of the floxed and excised (exon 2) allele ( $\triangle E 2)$ of Cthrc1 and the presence of Osx1-Cre. Genomic DNA was extracted from femurs after bone marrow cells were flashed out, and genomic PCR analysis was performed. (B) Cthrc1 deletion in osteoblasts did not affect Cthrc1 expression at baseline or after RANKL injections. GST-RANKL was injected into $C$ thrc $1^{f / f l}$ control or $\triangle \mathrm{OB}$ mice. RNAs from long bone were extracted 3 days after 2 RANKL injections, and real-time RT-PCR analysis was performed for Cthrc1, Acp5, Calcr, and Ctsk expression. $n=4$ per group. (C) $\mu$ CT analysis of the trabecular region of the proximal tibial metaphysis of Cthrc $1^{f l f f l}$ control and $\Delta O B$ mice. (D) Representative $\mu \mathrm{CT}$ images of the proximal tibia of control and $\triangle \mathrm{OB}$ mice from C. Scale bars: $1 \mathrm{~mm} .{ }^{\star} P<0.05 ;{ }^{* \star} P<0.01 ;{ }^{* \star \star} P<0.001$.

cess induced acute bone loss, reaching a nadir in $3 \mathrm{D}$ BV at 2 weeks that was followed by a gradual recovery of bone mass due to stimulation of bone formation. In $\triangle \mathrm{OC}$ mice, the initial catabolic phase was indistinguishable from that of the control group, with the same extent of bone loss, which indicates that CTHRC1 deficiency in osteoclasts has no major effect on the bone resorption response to acute RANKL challenge. However, subsequent recovery of bone mass was significantly delayed in the absence of CTHRC1, which suggests that the relay signal from bone resorption to formation is impaired when CTHRC1 production is abrogated in osteoclasts. Thus, we propose that CTHRC1 is a physiologically important osteoclast-produced signal that is relayed to bone formation (Supplemental Figure 9).

The reduced $C t h r c 1$ expression that occurs with aging and after alendronate treatment in vivo is thought to dampen the coupling process and leads to insufficient new bone replacement at remodeling sites (Supplemental Figure 9). Consistent with this concept is a previous report showing that forced Cthrc1 expression in osteoblasts results in a high bone mass phenotype with stimulated bone formation (14). Thus, although further studies are required to clarify its causative role in age-related bone loss, CTHRC1 may provide an attractive target in the diagnosis and treatment of bone diseases such as osteoporosis, which is considered a coupling disorder in the bony skeleton.

CTHRC1 was originally identified in the arterial wall, where its gene expression was induced only when the carotid artery and aorta were injured (11). In addition to being responsive to TGF- $\beta 1$, CTHRC1 is proposed to contribute to vascular remodeling by regulating matrix deposition and cell migration. During development, CTHRC1 is expressed in the node/notochord (16) and then the growth plate cartilage (refs. 13, 14, and the present study). However, no apparent abnormality in skeletal development has been observed in mice lacking Cthrc1 (refs. 14, 16, and the present study), so the physiologic function of CTHRC1 in development is not yet clear. During adulthood, in contrast, basal Cthrc1 expression is highly restricted to bone and brain (ref. 11 and the present study). In bone, Cthrc1 was predominantly expressed in osteoclasts, which was also supported by quantitative RT-PCR. These results showed that Cthrc1 expression in $\triangle \mathrm{OC}$ mouse bone was markedly reduced, in fact, to the same level as in global Cthrc1 KO mice (present study). We speculate that in postnatal life, CTHRC1 functions in skeletal as well as vascular remodeling, with dynamic vascular remodeling taking place in response to injury, when Cthrc1 is transiently induced. In this manner, bone is continuously remodeled: bone-resorbing osteoclasts actively produce and secrete CTHRC1 in order to promote bone remodeling.

\section{Methods}

Reagents, recombinant proteins, and antibodies. Alendronate sodium hydrate was from Teijin Pharma Ltd.; bafilomycin A1, gadolinium chloride hexahydrate, N-ethylmaleimide, dexamethasone, 3-isobutyl-1-methylxanthine, and salmon calcitonin were from Sigma-Aldrich; recombinant BMP-2 and FGF-1 were from R\&D Systems Inc.; and Osteologic discs were from BD Biosciences.

Recombinant murine M-CSF and GST-RANKL were expressed as described previously (30). Recombinant mouse CTHRC1 proteins were generated using the baculovirus/insect cell system according to standard 

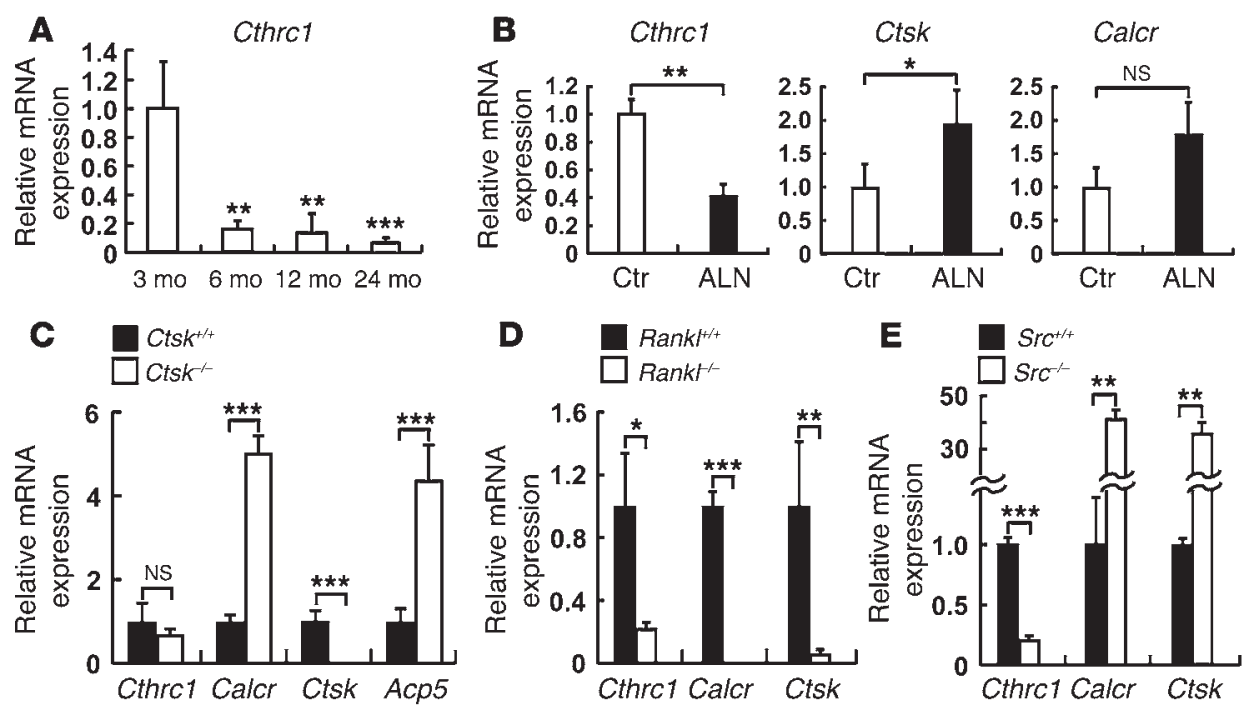

\section{Figure 8}

Correlation of Cthrc1 expression with bone turnover states. (A) Cthrc1 expression declined with aging. Cthrc 1 mRNA expression in bone was quantitated among male mice of the indicated ages by quantitative RT-PCR. $n=3$. (B) Inhibition of Cthrc1 expression after alendronate treatment in vivo. (C-E) Cthrc1 expression was examined in bone from $\mathrm{Ctsk}^{-/-}$ (C), Rankl/- (D), and Src ${ }^{-/-}$(E) osteopetrotic mouse models. Expression of osteoclast marker genes Calcr, Ctsk, and Acp5 was also determined. $n=3$. ${ }^{\star} P<0.05 ;{ }^{*} P<0.01 ;{ }^{* \star *} P<0.001$. procedures. In brief, mouse Cthrc1 cDNA containing a His-tag in a pPSC8 vector (Protein Science) was cotransfected with AcNPV DNA (Invitrogen) using CELLFECTIN (Invitrogen) into Sf9 cells, which were then infected into expresSF+ ${ }^{+}$insect cells (Protein Sciences) using recombinant viruses. Cell supernatant was harvested, and the protein was purified using $\mathrm{Ni}$ Sepharose High Performance (Amersham Biosciences) and a Tricon 10/20 Column (Amersham Biosciences).

A polyclonal antibody against recombinant His-tagged murine CTHRC1 protein was raised with a standard technique and affinity purified using a CTHRC1 protein-conjugated sepharose column. A mouse monoclonal antibody against mouse CTHRC1, 13B1, was established according to standard procedures.

Isolation of BMMs and osteoclastogenesis. BMMs were prepared from whole bone marrow of 8- to 10-week-old C57BL/6 mice (Clea Japan Inc.) and cultured as described previously (31).

TRAP-positive mononuclear pOCs were prepared from a 2-day culture of BMMs in $\alpha \mathrm{MEM}$ containing 10\% FBS in the presence of M-CSF and RANKL. These pOCs were further cultured in the presence of M-CSF and RANKL to generate either inactive mature osteoclasts prepared on plastic dishes or active mature osteoclasts prepared on dentin slices.

RNA isolation and RT-PCR. Total RNAs were extracted from cells using TRIzol reagent (Invitrogen) and used for microarray and RT-PCR analyses. See Supplemental Table 1 for primers used for RT-PCR. Relative gene expression was determined by SYBR green-based real-time PCR using a 7300 fast real-time PCR system (Applied Biosystems). Expression levels were normalized to Gapdh.

Microarray analysis. Total RNAs were processed according to the protocol recommended by Affymetrix. $5 \mu \mathrm{g}$ labeled cRNA from each sample was hybridized to the mouse genome 430 2.0 Array GeneChip (Affymetrix) representing approximately 38,500 genes. GeneChip data output was normalized and analyzed using Affymetrix GeneChip Operating Software version 1.1. Raw GeneChip data were transformed into log format following the recommendation by Affymetrix. Microarray data were deposited in GEO (accession no. GSE45656).

Immunoblotting. Immunoblotting was performed as described previously (32). Culture supernatants of BMMs and of mature osteoclasts prepared on either plastic dishes or dentin slices were immunoprecipitated with an anti-CTHRC1 monoclonal antibody, 13B1, and protein A/G agarose (SantaCruz). Samples were boiled in SDS sample buffer containing 2-mercaptoethanol and subjected to electrophoresis on $12 \%$ SDS-PAGE. Proteins were transferred to nitrocellulose membranes using a semidry blotter (BioRad) and incubated in blocking solution (5\% nonfat dry milk in TBS containing $0.1 \%$ Tween 20 ) for 1 hour to reduce nonspecific binding. Membranes were then exposed to rabbit anti-CTHRC1 polyclonal antibody overnight at $4^{\circ} \mathrm{C}$, washed 3 times, and incubated with secondary rabbit IgG horseradish peroxidase-conjugated antibody for 1 hour. Membranes were washed extensively, and enhanced chemiluminescence detection assay was performed according to the manufacturer's instructions.

$I S H$. The femur and tibia from 3-week-old C57BL/6 male mice were dissected, fixed, decalcified, embedded in paraffin, and sectioned at $4 \mu \mathrm{m}$. Paraffin sections were stained histochemically for TRAP in order to visualize osteoclasts.

For ISH, tissue sections were dewaxed with xylene and rehydrated through an ethanol series and PBS. Sections were fixed in $4 \%$ paraformaldehyde in PBS for 15 minutes, then washed with PBS. Next, the sections were treated with $7 \mu \mathrm{g} / \mathrm{ml}$ proteinase $\mathrm{K}$ in PBS for 30 minutes at $37^{\circ} \mathrm{C}$, washed with PBS, refixed with $4 \%$ paraformaldehyde in PBS, again washed with PBS, and placed in $0.2 \mathrm{~N} \mathrm{HCl}$ for 10 minutes. After washing with PBS, sections were acetylated by incubation in $0.1 \mathrm{M}$ tri-ethanolamine- $\mathrm{HCl}(\mathrm{pH} 8.0), 0.25 \%$ acetic anhydride, for 10 minutes. After washing with PBS, sections were dehydrated through a series of ethanol solutions. Hybridization was performed with probes at concentrations of $300 \mathrm{ng} / \mathrm{ml}$ in Probe Diluent-1 (Genostaff) at $60^{\circ} \mathrm{C}$ for 16 hours. After hybridization, sections were washed in $5 \times$ HybriWash (equal to $5 \times \mathrm{SSC}$ ) at $50^{\circ} \mathrm{C}$ for 20 minutes, then in $50 \%$ formamide with $2 \times$ HybriWash at $50^{\circ} \mathrm{C}$ for 20 minutes, followed by RNase treatment with $50 \mu \mathrm{g} / \mathrm{ml}$ RNase A in $10 \mathrm{mM}$ Tris- $\mathrm{HCl}$ (pH 8.0), $1 \mathrm{M} \mathrm{NaCl}$, and $1 \mathrm{mM}$ EDTA for 30 minutes at $37^{\circ} \mathrm{C}$. Sections were then washed twice with $2 \times$ HybriWash at $50^{\circ} \mathrm{C}$ for 20 minutes, twice with $0.2 \times$ HybriWash at $50^{\circ} \mathrm{C}$ for 20 minutes, and once with TBST (0.1\% Tween 20 in TBS). After treatment with $0.5 \%$ blocking reagent (Roche) in TBST for 30 minutes, sections were incubated for 2 hours at room temperature with anti-DIG AP conjugate (Roche) diluted 1:1,000 with TBST. Sections were washed twice with TBST and then incubated in $100 \mathrm{mM} \mathrm{NaCl}, 50 \mathrm{mM} \mathrm{MgCl}_{2}$, and $0.1 \%$ Tween 20 in $100 \mathrm{mM}$ Tris- $\mathrm{HCl}$ ( $\mathrm{pH}$ 9.5). Coloring reactions were performed with NBT/ BCIP solution (Sigma-Aldrich) overnight, followed by washing with PBS. Sections were counterstained with Kernechtrot stain solution (Mutoh), dehydrated, and mounted with Malinol (Mutoh).

Retroviral expression. The retroviral vector pMX-puro with Cthrc1 cDNA and/or the pVSVG plasmid were used to transfect the retrovirus packaging cells, Plat-E (gift of T. Kitamura, University of Tokyo, Tokyo, Japan) or 
GP2-293 (Clontech Co. Ltd.). Calvaria-derived primary osteoblasts and preadipocytic 3T3-L1 cells were infected with the retroviral vector PMX-puro expressing CTHRC1 under the control of Mo-MLV LTR, then cultured in the presence of polybrene $(8 \mu \mathrm{g} / \mathrm{ml})$ for 1 day. Infected cells were cultured in the presence of $2 \mu \mathrm{g} / \mathrm{ml}$ puromycin, and ALP and alizarin red staining was performed as described previously (33). Transduced 3T3-L1 cells were cultured in the presence of IBMX and dexamethasone to induce adipogenesis and were stained with Oil Red-O.

Flow cytometry. ST2 cells were reacted with or without recombinant CTHRC1 protein $(10 \mu \mathrm{g} / \mathrm{ml})$ in PBS for 15 minutes. After washing, cells were stained with rabbit anti-CTHRC1 polyclonal antibody or rabbit IgG as a negative control, and PE-conjugated goat anti-rabbit IgG (Jackson), then analyzed using flow cytometry (FACSCalibur; Becton Dickinson).

Bone formation assay. Organ cultures of mouse calvaria were performed as described previously (34). Briefly, calvariae from 4-day-old pups of C57BL/6 mice were explanted, dissected free of any adjacent connective tissue, placed in BGJ media (Sigma-Aldrich) containing $0.1 \%$ BSA (Sigma-Aldrich), and incubated with recombinant CTHRC1 (20 or $200 \mathrm{ng} / \mathrm{ml}$ ) for 7 days. Recombinant human BMP-2 (100 ng/ml) or FGF-1 $(100 \mathrm{ng} / \mathrm{ml})$ was added as a positive control. Media were changed every 3 days. Calvariae were then fixed, decalcified, embedded in paraffin, and sectioned at $4 \mu \mathrm{m}$. Sections were placed on coated glass slides and stained with hematoxylin and eosin. Bone-forming activity was assessed by measuring calvaria thickness.

In vitro chemotactic assay. EZ-TAXIScan (Effector Cell Institute) was used to detect real-time horizontal chemotaxis of ST2 cells. The EZ-TAXIScan consists of an etched silicon substrate and a flat glass plate, which form 2 compartments with a 5 - $\mu$ m-deep microchannel. In some experiments, Thermanox coverslips (Nalgen Nunc International) were placed onto the glass plates. ST2 cells were placed into the single hole with which the device is held together of the stainless steel holder, and $1 \mu \mathrm{l}$ recombinant CTHRC1 protein and/or WNT3A (R\&D) was placed into the contrahole. A charge-coupled device (CCD) camera was used to record the migration of ST2 cells toward the CTHRC1 and/or WNT3A on the microchannel. Moving cells in a fixed gate were counted using a TAXIScan Analyzer (Effector Cell Institute).

Chemotaxis of ST2 cells or calvaria-derived osteoblasts was also assessed using the Cultrex 96 Well Cell Migration Assay Kit (Trevigen Inc.). In brief, stromal cells at $80 \%$ confluence were serum starved in $0.5 \% \mathrm{FBS}$ for 24 hours. Cells were harvested, and $5 \times 10^{4}$ cells were added to the top chamber. Either CTHRC1, WNT3A, or both was added to the bottom chamber, and cells were cultured for 24 hours. After a washing of each well, the migrating cells were stained with calcein and examined with a reader plate at $485 \mathrm{~nm}$ excitation, $520 \mathrm{~nm}$ emission.

$\mathrm{KO}$ mouse generation and animal experiments. A genomic fragment containing Cthrc1 was obtained from the BAC genomic library of the mouse 129SvEv strain and targeted by homologous recombination in ES cells established from blastocysts of BA1 Hybrid (C57BL/6 × 129SvEv) mice. The targeting construct introduced 3 loxP sites into the gene flanking exon 2, which encodes the collagen domain, and the neo cassette was also flanked by 2 FRT sites (Figure 4A). ES cells with a targeted allele were microinjected into blastocysts derived from $\mathrm{C} 57 \mathrm{BL} / 6$ mice, and the germline chimeras generated by the blastocyst injections were crossed with C57BL/ 6 female mice. $2 \mathrm{~F} 1$ heterozygous mice were born and mated with Flp mice to delete the neo cassette. Mice were backcrossed 6 generations onto the C57BL/6J genetic background.

The generation of Ctsk-Cre (Ctsk $\left.\mathrm{Cre} /+^{+}\right)$mice (provided by T. Nakamura, Keio University, Tokyo, Japan) has been described previously (17). To disrupt Cthrc1 specifically in mature osteoclasts or osteoblasts, the Ctsk ${ }^{\mathrm{Cr} / \mathrm{+}}$ knockin

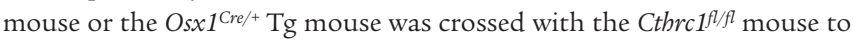

generate $C t s k^{C r e /+} C t h r c 1^{\triangle O C / \triangle O C}(\Delta \mathrm{OC})$ or $O s \times 1^{\mathrm{Cre} /+} C t h r c 1^{\triangle O B / \triangle O B}(\triangle \mathrm{OB})$ mice. Cthrc $1^{l / f l}$ mice were used as controls. CAG-Cre mice were obtained from M. Okabe (Osaka University, Osaka, Japan) and crossed with Cthrc fl/fl $^{\text {mice }}$ to generate $\mathrm{Cthrc1}^{+/+}, \mathrm{Cthrc1}^{+/-}$, and $\mathrm{Cthrc1}^{-/-}(\mathrm{sKO})$ mice. Rankll/fl mice were generated by inserting a LoxP-FRT-PGKneo-FRT cassette into intron 2 and another LoxP site into intron 4 of Rankl, so that exons 3 and 4 of Rankl would be deleted on crossing with tissue-specific Cre transgenic mice (our unpublished observations). Rankl ${ }^{-/-}$mice were obtained by crossing Rankll/fl and $\mathrm{CAG}$-Cre mice. $\mathrm{Src}^{-1-}$ mice were obtained from the Jackson Laboratory.

Mice were raised under standard laboratory conditions at $24^{\circ} \mathrm{C} \pm 2{ }^{\circ} \mathrm{C}$ and 50\% 60\% humidity, and allowed free access to tap water and commercial standard rodent chow (CE-2) containing $1.20 \%$ calcium, $1.08 \%$ phosphate, and $240 \mathrm{IU} / 100 \mathrm{~g}$ vitamin $\mathrm{D}_{3}$ (Clea Japan Inc.). Wild-type C57BL/6 or transgenic mice were treated s.c. with vehicle (saline) or $1 \mathrm{mg} / \mathrm{kg} \mathrm{BW}$ alendronate 5 times per week for 3 weeks. Wild-type or $\triangle \mathrm{OC}$ mice were treated with $1 \mathrm{mg} / \mathrm{kg}$ BW GST-RANKL twice with a 24-hour interval. Blood samples at sacrifice were centrifuged to obtain the serum.

Bone analysis. Bone histomorphometry was performed on undecalcified sections, with tetracycline and calcein double labeling. The histomorphometric parameters were measured at the Niigata Bone Science Institute.

$\mu C T$ scanning was performed on proximal tibiae using a $\mu C T-40$ scanner (SCANCO Medical AG) with a resolution of $12 \mu \mathrm{m}$, and 3D microstructure parameters were calculated as described previously (35). The proximal tibia was positioned so as to be scanned craniocaudally using 320 slices with $12-\mu \mathrm{m}$ increments at $45 \mathrm{kVp}$ and $177 \mu \mathrm{A}$. On the original 3D image, morphometric indices - including BV, tissue volume, trabecular thickness, trabecular separation, and trabecular number - were directly determined from the binarized volume of interest (VOI). Nonmetric parameters, such as the structure model index (SMI) and connectivity density, were also obtained as described previously (35); for SMI, the characteristic form of a 3-dimensionally described structure in terms of the amount of plates and rods composing the structure was quantified, and connectivity density was determined as the number of trabecular connections per cubic millimeter. The nomenclature for $\mu \mathrm{CT}$ and histomorphometry followed previously published guidelines $(36,37)$.

Biochemical analysis. CTX concentrations in serum and urine were determined using a RatLaps assay according to the manufacturer's protocols (Immunodiagnostic Systems Ltd.). Serum osteocalcin concentration was measured using a mouse ostocalcin EIA kit (Biomedical Technologies Inc.).

Statistics. Data are expressed as mean \pm SD. Statistical analysis was performed using paired or unpaired Student's $t$ test (2-sample, 2-tailed comparison) or ANOVA followed by Dunnett test or Student-Newman-Keuls test. A $P$ value less than 0.05 was considered significant.

Study approval. All experiments were performed in accordance with NCGG ethical guidelines for animal care, and experimental protocols were approved by the animal care committee.

\section{Acknowledgments}

We thank Mie Suzuki for technical assistance, Akemi Ito (Ito Bone Science Institute) for valuable suggestions on bone histomorphometry, Masaru Okabe for CAG-Cre mice, Takashi Nakamura for Ctsk-Cre mice, Hiroko Meguro and Shogo Yamamoto (University of Tokyo) for microarray analysis, and members of NCGG for stimulating discussions. This study was supported in part by a Grant-in-aid for Scientific Research C (no. 18590359; to S. Takeshita) and a Grant-in-aid for Scientific Research on Innovative Areas (no. 22118007; to K. Ikeda) from the Ministry of Education, Science of Japan; by a grant from Promotion of Fundamental Studies in Health Sciences of the National Institute of Biomedical Innovation (NIBIO) of Japan (06-31; to K. Ikeda); and by grants from Ono Foundation (to S. Takeshita), Daiko Foundation (to 
K. Ikeda), and Tokyo Biochemistry Research Foundation (to K. Ikeda). Pacific Edit reviewed the manuscript prior to submission.

Received for publication February 22, 2013, and accepted in revised form May 31, 2013.

1. Zaidi M. Skeletal remodeling in health and disease. Nat Med. 2007;13(7):791-801.

2. Karsenty G, Wagner EF. Reaching a genetic and molecular understanding of skeletal development. Dev Cell. 2002;2(4):389-406.

3. Hattner R, Epker BN, Frost HM. Suggested sequential mode of control of changes in cell behaviour in adult bone remodelling. Nature. 1965; 206(983):489-490.

4. Black DM, et al. The effects of parathyroid hormone and alendronate alone or in combination in postmenopausal osteoporosis. N Engl J Med. 2003; 349:1207-1215.

5. Martin TJ, Sims NA. Osteoclast-derived activity in the coupling of bone formation to resorption. Trends Mol Med. 2005;11(2):76-81.

6 . Tang Y, et al. TGF-beta1-induced migration of bone mesenchymal stem cells couples bone resorption with formation. Nat Med. 2009;15(7):757-765.

7. Zhao C, et al. Bidirectional ephrinB2-EphB4 signaling controls bone homeostasis. Cell Metab. 2006; 4(2):111-121.

8. Ryu J, Kim HJ, Chang EJ, Huang H, Banno Y, Kim $\mathrm{HH}$. Sphingosine 1-phosphate as a regulator of osteoclast differentiation and osteoclast-osteoblast coupling. EMBO J. 2006;25(24):5840-5851.

9. Pederson L, Ruan M, Westendorf JJ, Khosla S, Oursler MJ. Regulation of bone formation by osteoclasts involves Wnt/BMP signaling and the chemokine sphingosine-1-phosphate. Proc Natl Acad SciU S A. 2008;105(52):20764-20769.

10. Lotinun S, et al. Osteoclast-specific cathepsin K deletion stimulates S1P-dependent bone formation. J Clin Invest. 2013;123(2):666-681.

11. Pyagay P, et al. Collagen triple helix repeat containing 1 , a novel secreted protein in injured and diseased arteries, inhibits collagen expression and promotes cell migration. Circ Res. 2005;96(2):261-268

12. LeClair R, Lindner V. The role of collagen triple helix repeat containing 1 in injured arteries, collagen expression, and transforming growth factor beta signaling. Trends Cardiovasc Med. 2007; 17(6):202-205.

13. Durmus T, LeClair RJ, Park KS, Terzic A, Yoon JK,

Address correspondence to: Sunao Takeshita, Department of Bone and Joint Disease, National Center for Geriatrics and Gerontology (NCGG), 35 Gengo, Morioka, Obu, Aichi 474-8511, Japan. Phone: 81.562.44.5651, ext. 5047; Fax: 81.562.44.6595; E-mail: sunao@ncgg.go.jp.

Lindner V. Expression analysis of the novel gene collagen triple helix repeat containing-1 (Cthrc1). Gene Expr Patterns. 2006;6(8):935-940.

14. Kimura $\mathrm{H}$, et al. Cthrc1 is a positive regulator of osteoblastic bone formation. PLoS One. 2008;3(9):e3174.

15. Kadowaki T, Yamauchi T. Adiponectin and adiponectin receptors. Endocr Rev. 2005;26:439-451.

16. Yamamoto $S$, et al. Cthrc1 selectively activates the planar cell polarity pathway of Wnt signaling by stabilizing the Wnt-receptor complex. Dev Cell. 2008; 15(1):23-36.

17. Nakamura T, et al. Estrogen prevents bone loss via estrogen receptor alpha and induction of Fas ligand in osteoclasts. Cell. 2007;130(5):811-823.

18. Matsumura $\mathrm{H}$, Hasuwa $\mathrm{H}$, Inoue $\mathrm{N}$, Ikawa $\mathrm{M}$, Okabe M. Lineage-specific cell disruption in living mice by Cre-mediated expression of diphtheria toxin A chain. Biochem Biophys Res Commun. 2004; 321(2):275-279.

19. Tomimori Y, et al. Evaluation of pharmaceuticals with a novel 50-hour animal model of bone loss. J Bone Miner Res. 2009;24(7):1194-1205.

20. Rodda SJ, McMahon AP. Distinct roles for Hedgehog and canonical Wnt signaling in specification, differentiation and maintenance of osteoblast progenitors. Development. 2006;133(16):3231-3244.

21. Wu X, et al. Inhibition of Sca-1-positive skeletal stem cell recruitment by alendronate blunts the anabolic effects of parathyroid hormone on bone remodeling. Cell Stem Cell. 2010;7(5):571-580.

22. Khosla S. Odanacatib: location and timing are everything. J Bone Miner Res. 2012;27(3):506-508.

23. Pennypacker B, et al. Bone density, strength, and formation in adult cathepsin $\mathrm{K}(-/-)$ mice. Bone. 2009;44(2):199-207.

24. Henriksen K, Bollerslev J, Everts V, Karsdal MA. Osteoclast activity and subtypes as a function of physiology and pathology - implications for future treatments of osteoporosis. Endocr Rev. 2011; 32(1):31-63.

25. Takahashi H, Epker B, Frost HM. Resorption precedes formative activity. Surg Forum. 1964; 15:437-438.

26. Howard GA, Bottemiller BL, Turner RT, Rader JI,
Baylink DJ. Parathyroid hormone stimulates bone formation and resorption in organ culture: evidence for a coupling mechanism. Proc Natl Acad Sci US A. 1981;78(5):3204-3208.

27. Karsdal MA, et al. Acidification of the osteoclastic resorption compartment provides insight into the coupling of bone formation to bone resorption. Am J Pathol. 2005;166(2):467-476.

28. Lee SH, et al. v-ATPase V0 subunit d2-deficient mice exhibit impaired osteoclast fusion and increased bone formation. Nat Med. 2006;12(12):1403-1409.

29. Ishii M, et al. Sphingosine-1-phosphate mobilizes osteoclast precursors and regulates bone homeostasis. Nature. 2009;458(7237):524-528.

30. Takeshita S, et al. SHIP-deficient mice are severely osteoporotic due to increased numbers of hyperresorptive osteoclasts. Nat Med. 2002;8(9):943-949.

31. Takeshita S, Kaji K, Kudo A. Identification and characterization of the new osteoclast progenitor with macrophage phenotypes being able to differentiate into mature osteoclasts. J Bone Miner Res. 2000; 15(8):1477-1488.

32. Ishii KA, et al. Coordination of PGC-1beta and iron uptake in mitochondrial biogenesis and osteoclast activation. Nat Med. 2009;15(3):259-266.

33. Tatsumi S, et al. Targeted ablation of osteocytes induces osteoporosis with defective mechanotransduction. Cell Metab. 2007;5(6):464-475.

34. Mundy G, et al. Stimulation of bone formation in vitro and in rodents by statins. Science. 1999; 286(5446):1946-1949.

35. Ito $\mathrm{M}$, et al. Multi-detector row CT imaging of vertebral microstructure for evaluation of fracture risk. J Bone Miner Res. 2005;20(10):1828-1836.

36. Bouxsein ML, Boyd SK, Christiansen BA, Guldberg RE, Jepsen KJ, Muller R. Guidelines for assessment of bone microstructure in rodents using microcomputed tomography. J Bone Miner Res. 2010; 25(7):1468-1486.

37. Dempster DW, et al. Standardized nomenclature, symbols, and units for bone histomorphometry: a 2012 update of the report of the ASBMR Histomorphometry Nomenclature Committee. J Bone Miner Res. 2013;28(1):2-17. 